\title{
Epithelial dysplasia of the stomach with gastric immunophenotype shows features of biological aggressiveness
}

\author{
Pedro Valente $\cdot$ Mónica Garrido $\cdot$ Irene Gullo $\cdot$ Helena Baldaia • \\ Margarida Marques $\cdot$ Francisco Baldaque-Silva • \\ Joanne Lopes · Fátima Carneiro
}

Received: 6 February 2014 / Accepted: 28 July 2014/Published online: 22 August 2014

(c) The International Gastric Cancer Association and The Japanese Gastric Cancer Association 2014

\begin{abstract}
Background Gastric dysplasia is classified as adenomatous/type I (intestinal phenotype) and foveolar or pyloric/ type II (gastric phenotype) according to morphological (architectural and cytological) features. The immunophenotypic classification of dysplasia, based on the expression of the mucins, CD10 and CDX2, recognizes the following immunophenotypes: intestinal (MUC2, CD10, and CDX2); gastric (MUC5AC and/or MUC6, absence of CD10, and absent or low expression of CDX2); hybrid (gastric and intestinal markers); and null.
\end{abstract}

P. Valente and M. Garrido contributed equally.

P. Valente · M. Garrido · I. Gullo · F. Carneiro Faculty of Medicine of the University of Porto, Porto, Portugal e-mail: pedrofmvalente@gmail.com

I. Gullo

Faculty of Medicine and Surgery, University of Genova, Genova, Italy

H. Baldaia - M. Marques - F. Baldaque-Silva $\cdot$ J. Lopes ·

F. Carneiro

Centro Hospitalar de São João, Porto, Portugal

F. Baldaque-Silva

Department of Gastroenterology and Hepatology, Karolinska

University Hospital, Stockholm, Sweden

F. Carneiro $(\square)$

Institute of Molecular Pathology and Immunology,

University of Porto (IPATIMUP), Rua Dr. Roberto Frias S/N,

4200-465 Porto, Portugal

e-mail: fcarneiro@ipatimup.pt
Methods Sixty-six cases of nonpolypoid epithelial dysplasia of the stomach were classified according to morphological features (histotype and grade) and immunophenotype. Immunohistochemical staining was performed with antibodies against MUC2, MUC5AC, MUC6, CD10, CDX2, chromogranin, synaptophysin, Ki-67, and TP53. HER2 alterations were analyzed by immunohistochemistry and silver-enhanced in situ hybridization.

Results By conventional histology, dysplasia was classified as adenomatous/intestinal $(n=42 ; 64 \%)$ and foveolar or pyloric/gastric $(n=24 ; 36 \%)$ and graded as low grade $(n=37 ; 56 \%)$ or high grade $(n=29 ; 44 \%)$. Immunophenotypic classification showed intestinal $(n=22$; $33.3 \%)$, gastric $(n=25 ; 37.9 \%)$, hybrid $(n=17$; $25.8 \%)$, or null $(n=2 ; 3.0 \%)$ phenotypes. In 20 cases a coexistent intramucosal carcinoma was identified. The intestinal immunophenotype was shown to be significantly associated with low-grade dysplasia $(p=0.001)$, high expression of CDX2 $(p=0.015)$, TP53 $(p=0.034)$, synaptophysin $(p=0.003)$, and chromogranin $(p<0.0001)$; the gastric immunophenotype was significantly associated with high-grade dysplasia $(p=0.001)$, high Ki-67 proliferative index $(p=0.05)$, and coexistence of intramucosal carcinoma $(p=0.013)$. HER 2 amplification was observed in 3 cases, typed as gastric or hybrid.

Conclusions Epithelial nonpolypoid dysplasia of the stomach with gastric immunophenotype shows features of biological aggressiveness and may represent the putative precursor lesion in a pathway of gastric carcinogenesis originated de novo from the native gastric mucosa, leading to gastric-type adenocarcinoma.

Keywords Gastric carcinogenesis - Dysplasia - HER2 . Immunophenotype $\cdot$ Mucins 


\section{Introduction}

At present, gastric carcinoma (GC) has significant morbidity/mortality impact, being the fourth most common cancer worldwide and the second most deadly [1].

According to Laurén's [2] classification, there are two main subtypes of GC, intestinal and diffuse, which differ in epidemiology, pathogenesis, morphology, and molecular features [2,3]. According to the Correa [4] model, gastric cancer develops along a cascade of lesions encompassing Helicobacter pylori-induced chronic superficial gastritis, chronic atrophic gastritis, intestinal metaplasia, dysplasia, and ultimately invasive adenocarcinoma. Gastric dysplasia is neoplastic in nature and is a direct precursor of GC, as well as a risk factor of carcinoma in other locations of the stomach [5, 6].

Dysplasia is graded as low- or high grade on the basis of architectural and cell features. Further, according to the histomorphological profile, dysplasia may be classified as adenomatous/type I (intestinal phenotype) and foveolar or pyloric/type II (gastric phenotype). The two types may be distinguished by the immunoexpression of the mucins, CD10 and CDX2 (intestinal/adenomatous: MUC2, CD10, and CDX2; gastric/foveolar: MUC5AC and/or MUC6, absence of CD10, and low or absent expression of CDX2) [7-9]. Cases with hybrid differentiation may also occur as well as null cases in which there is no expression of the aforementioned markers [8].

A relationship has been reported between the histological grade and the immunohistochemical profiles of dysplasia: in one study, $81.8 \%$ of low-grade dysplasia expressed intestinal markers, and $72.2 \%$ of high-grade dysplasia showed markers of gastric differentiation with variable expression of intestinal markers [10]. In another study, foveolar and hybrid subtypes were also significantly associated with high-grade dysplasia [8].

In this study we aimed at analyzing the relationship between different types of gastric dysplasia (based on histotypes and grading) and the immunohistochemical profile according to the expression of markers of cell differentiation (MUC5AC, MUC6, MUC2, CD10). The expression of CDX2, Ki-67, TP53, HER2, and neuroendocrine markers (chromogranin and synaptophysin) was also evaluated.

\section{Materials and methods}

A series of 66 cases of epithelial dysplasia of the stomach identified in endoscopic submucosal dissection (ESD) specimens were retrieved retrospectively from the files of the Department of Pathology, Centro Hospitalar São João, between June 2010 and June 2013. The cases encompassed superficial nonpolypoid dysplastic lesions (0-II), including slightly elevated (0-IIa), flat (0-IIb), and slightly depressed (0-IIc) lesions, according to the Paris classification [11]. In 20 cases a coexistent intramucosal carcinoma was identified. The study was approved by the Ethical Committee of the Hospital. The clinicopathological features of the cases are summarized in Table 1.

Tissues were fixed in neutral buffered $10 \%$ formalin, embedded in paraffin, and cut into 3- $\mu \mathrm{m}$-thick sections.

The lesions were classified on hematoxylin and eosin (H\&E)-stained slides as adenomatous/type I (intestinal phenotype) and foveolar or pyloric/type II (gastric phenotype), according to the WHO classification [3]. The adenomatous/intestinal subtype resembles colonic adenomas, with crowded, tubular glands lined by atypical columnar cells with overlapping, pencillate, hyperchromatic and/or pleomorphic nuclei, with pseudostratification and mucin. The foveolar or pyloric/gastric phenotype is characterized by cuboid/low columnar cells, with round to oval nuclei and clear or eosinophilic cytoplasm.

The grade of the dysplasia was evaluated according to WHO 2010 criteria [3]. Low-grade dysplasia shows minimal architectural disarray and only mild to moderate cytological atypia; the nuclei are elongated/oval, polarized, and basally located and the mitotic activity is mild to moderate. High-grade dysplasia presents pronounced architectural disarray, such as complex branching or fusion of glands; the neoplastic cells are usually cuboidal, rather than columnar, with a high nuclear/cytoplasm ratio, high number of mitoses, occasionally atypical, and nuclei within the luminal zone of the epithelium with loss of polarity. The diagnosis of invasive carcinoma was performed when invasion of the lamina propria or muscularis mucosae occurred.

Immunohistochemical staining was performed with antibodies against MUC2, MUC5AC, MUC6, CD10, CDX2, chromogranin, synaptophysin, Ki-67, HER2, and TP53 (Table 2). Samples were processed in the automatic equipment Benchmark ULTRA using the Ultraview Universal DAB kit (Ventana Medical Systems, Roche Group). Each sample was heated and deparaffinized, followed by

Table 1 Clinicopathological features of the series of cases

\begin{tabular}{ll}
\hline Age (years, mean $\pm \mathrm{SD})$ & $65.95 \pm 10.93$ \\
Sex & \\
Male & 34 \\
Female & 32 \\
Tumor size (cm, mean $\pm \mathrm{SD})$ & $2.51 \pm 2.23$ \\
Location $^{\mathrm{a}}$ & \\
Body/fundus $^{\text {Antrum/pylorus }}$ & $20(31.3 \%)$ \\
\end{tabular}

${ }^{a}$ Missing data for location (2 cases) 
Table 2 Primary antibodies and immunohistochemistry conditions used in this study

\begin{tabular}{|c|c|c|c|c|c|c|}
\hline Antibody & Clone & $\begin{array}{l}\text { Antigen retrieval } \\
\text { conditions }\end{array}$ & Dilution & $\begin{array}{l}\text { Incubation } \\
\text { time }(\min ) \text { at } 37{ }^{\circ} \mathrm{C}\end{array}$ & Localization & Source \\
\hline $\mathrm{CDX} 2$ & EPR2764Y & $64 \min$ at $96^{\circ} \mathrm{C}$ & Pre-diluted & 28 & Nuclear & Cell Marque, USA \\
\hline MUC2 & Ccp58 & $52 \min$ at $96{ }^{\circ} \mathrm{C}$ & $1: 100$ & 36 & Cytoplasmatic & Novocastra, UK \\
\hline MUC5AC & MRQ-19 & $36 \min$ at $96^{\circ} \mathrm{C}$ & Pre-diluted & 24 & Cytoplasmatic & Cell Marque, USA \\
\hline MUC6 & MRQ-20 & $36 \min$ at $95^{\circ} \mathrm{C}$ & Pre-diluted & 28 & Cytoplasmatic & Cell Marque, USA \\
\hline CD10 & SP67 & $64 \min$ at $95^{\circ} \mathrm{C}$ & Pre-diluted & 40 & Membrane (brush border) & Ventana, USA \\
\hline Chromogranin & NS55 & $52 \mathrm{~min}$ at $96{ }^{\circ} \mathrm{C}$ & $1: 300$ & 36 & Cytoplasmatic & Invitrogen, USA \\
\hline Synaptophysin & SP11 & 36 min at $95{ }^{\circ} \mathrm{C}$ & $1: 150$ & 32 & Cytoplasmatic & Neomarkers, USA \\
\hline $\mathrm{Ki}-67$ & SP6 & $36 \min$ at $95^{\circ} \mathrm{C}$ & $1: 400$ & 32 & Nuclear & Neomarkers, USA \\
\hline HER2 & 4B5 & $36 \min$ at $95^{\circ} \mathrm{C}$ & Pre-diluted & 12 & Membrane & Ventana, USA \\
\hline TP53 & $318-6-11$ & $52 \min$ at $96{ }^{\circ} \mathrm{C}$ & $1: 200$ & 32 & Nuclear & DAKO, Denmark \\
\hline
\end{tabular}

Antigen retrieval performed with CC1 (Tris/borate/EDTA buffer with pH 8.4; Ventana Medical Systems, catalogue number 950-124)

antigen recovery through heat and high-pH buffer solution. Each primary antibody was incubated in an individually optimized time and temperature, followed by application of the detection system and contrast with hematoxylin and bluing reagent from the same manufacturer.

Immunoreactivity was scored as follows: the immunoexpression of MUC2, MUC5AC, MUC6, CD10, synaptophysin, and chromogranin was scored as positive when $\geq 5 \%$ of the dysplastic cells displayed immunoreactivity. HER2 immunoexpression was scored according to Rüschoff et al. [12]: 0, absence of immunoreactivity; $1+$, tumor cell cluster with faint or barely perceptible membrane reactivity irrespective of percentage of immunoreactive cells; $2+$, tumor cell cluster with weak to moderate (complete, lateral, or basolateral) reactivity irrespective of the percentage of immunoreactive cells; $3+$, tumor cell cluster with strong (complete, lateral, or basolateral) reactivity irrespective of the percentage of immunoreactive cells; for scoring purposes any nuclear or cytoplasmic background staining was disregarded. The immunoexpression of CDX2 was considered positive when $\geq 25 \%$ of the dysplastic cells displayed immunoreactivity [9]; immunoexpression of $\mathrm{Ki}-67$ and TP53 was classified as absent/low when immunoreactivity was displayed in $<50 \%$ of the dysplastic cells and high in the presence of $\geq 50 \%$ positive cells [13].

Detection of the number of copies of the HER2 gene was performed in the cases scored as $2+$ and $3+$ by immunohistochemistry with the silver-enhanced in situ hybridization (SISH) automated technique using the BenchMark XT equipment and the INFORM HER2 SISH probe (Ventana Medical Systems). The HER2/Chr17 ratio of each case was calculated using a minimum of 40 cells in two independent areas of dysplasia. Cases were assigned a score based on the guideline recommendations for HER2 testing in gastric cancer according to Rüschoff et al. [12]: negative, ratio
HER2:Cr17 $<2.0$ with $<4$ copies of HER2 gene; borderline, ratio HER2: $\mathrm{Cr} 17<2.0$ with $\geq 4$ and $<6$ copies of HER2 gene; positive, ratio HER2: $\mathrm{Cr} 17<2.0$ with $\geq 6$ copies of HER2 gene or ratio HER2:Cr17 $\geq 2.0$.

Statistical analysis

Appropriate statistical methods were used regarding the type of sample and its distribution. The data were analyzed with SPSS software v. 19.0 (SPSS Software, Chicago, IL, USA), using the chi-squared or Fischer's test. A $p$ value $<0.05$ was considered statistically significant.

\section{Results}

The study group was composed of 66 cases (Table 1), classified by conventional histology on H\&E-stained slides as foveolar or pyloric/gastric $(n=24 ; 36 \%)$ or adenomatous/intestinal $(n=42 ; 64 \%)$ (Fig. 1) and graded as low grade $(n=37 ; 56 \%)$ or high grade $(n=29 ; 44 \%)$. According to the immunophenotype, the cases were classified as gastric type $(n=25 ; 37.9 \%)$ (Fig. $2 \mathrm{a}, \mathrm{c}, \mathrm{e}, \mathrm{g}, \mathrm{i})$, intestinal type $(n=22 ; 33.3 \%)$ (Fig. $2 \mathrm{~b}, \mathrm{~d}, \mathrm{f}, \mathrm{h}, \mathrm{j})$, hybrid type $(n=17 ; 25.8 \%)$, or null $(n=2,3.0 \%)$. The latter were not considered for subsequent analysis.

Table 3 summarizes the expression of the different markers in the three immunophenotypes of gastric dysplasia. Statistically significant differences were observed between the immunophenotypes regarding the expression of MUC2 $(p=0.002)$, CD10 $(p<0.0001)$, MUC5AC $(p<0.0001)$, and MUC6 $(p<0.0001)$. Cases with low/ absent expression of CDX2 were observed only in the gastric immunophenotype $(p=0.015)$.

The frequency of cases with high expression of Ki-67 was significantly greater in the gastric and hybrid (84.0\% 

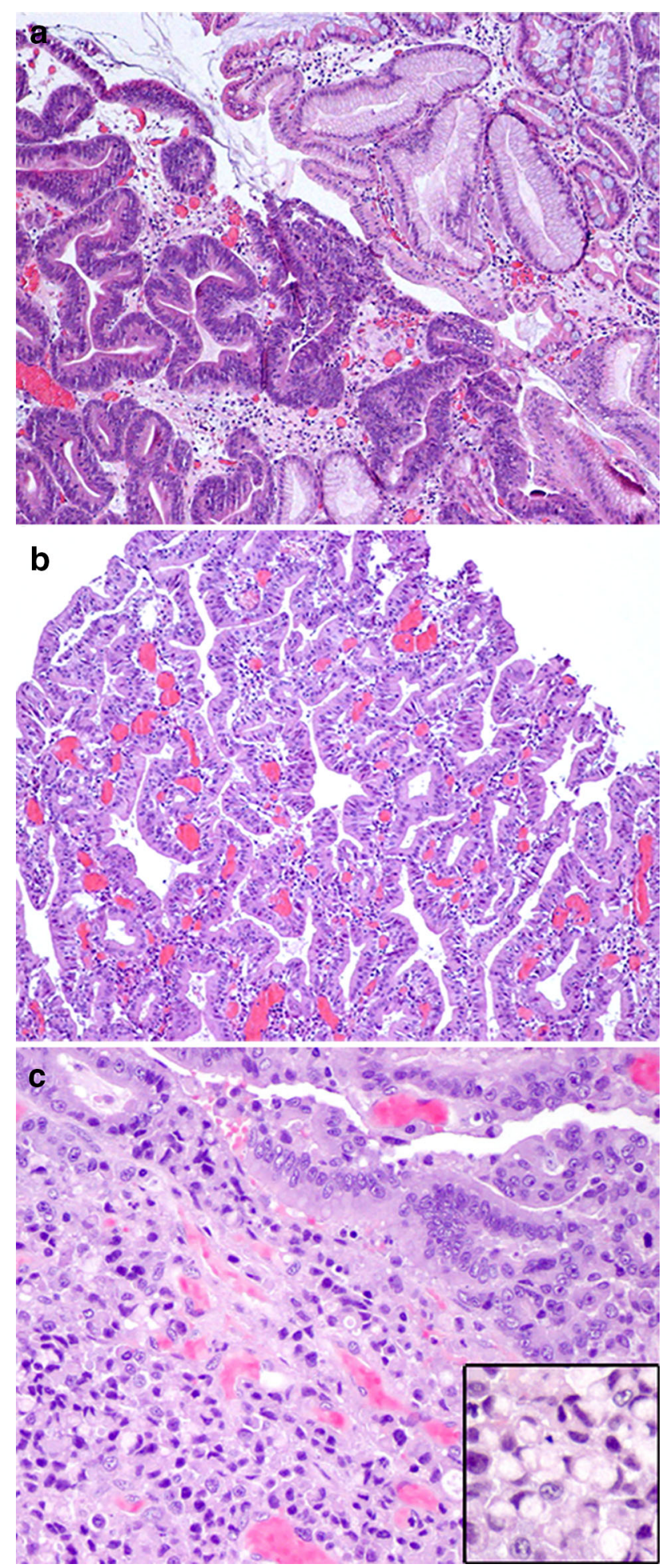

Fig. 1 Histotypes of gastric dysplasia. a Adenomatous/intestinal type, displaying tubular glands lined by columnar cells with overlapping, pencillated nuclei with pseudostratification. b Foveolar/gastric type, displaying cuboid/low columnar cells, with round to oval nuclei and eosinophilic cytoplasm. c Intramucosal carcinoma with signet ring cell component (inset) observed in the neighborhood of the dysplastic lesion shown in b. Hematoxylin and eosin (H\&E), $\times 100$ and $94.1 \%$, respectively) than in the intestinal $(63.6 \%)$ immunophenotypes $(p=0.05)$.

The frequency of cases with high expression of TP53 was significantly higher in the intestinal and hybrid (40.9\% and $52.9 \%$, respectively) than in the gastric $(16.0 \%)$ immunophenotypes $(p=0.034)$.

The expression of HER2 (Fig. 3a) was homogeneous and seen in areas of both dysplasia and adenocarcinoma. In dysplasia, scores $2+$ and $3+$ were observed in 11 lesions with gastric or hybrid immunophenotypes $(28.0 \%$ and $23.5 \%$, respectively) and not detected in the intestinal immunophenotype ( $p=0.029)$.

HER2 amplification (Fig. 3b) was observed in three cases of dysplasia, immunophenotyped as gastric $(n=1)$ and hybrid $(n=2)$, as well as in one adenocarcinoma identified in the neighborhood of a dysplastic lesion.

Regarding the neuroendocrine markers, the frequency of the expression of synaptophysin was significantly higher in intestinal $(81.8 \%)$ than in hybrid and gastric $(58.8 \%$ and $32.0 \%$, respectively) immunophenotypes $(p=0.003)$. Similar observations were made for the expression of chromogranin, displayed predominantly in intestinal $(95.2 \%)$ in comparison with hybrid and gastric $(70.6 \%$ and $28.0 \%$, respectively) immunophenotypes $(p<0.0001)$. In some cases, immunophenotyped as intestinal, small nests of neuroendocrine cells were observed.

Table 4 shows the relationship between the immunophenotypes and the histotypes of gastric dysplasia (adenomatous/intestinal and foveolar or pyloric/gastric), and grade (low- and high-grade). The frequency of high-grade dysplasia was significantly higher in the gastric immunophenotype $(68.0 \%)$ than in the other immunophenotypes (47.1\% and $13.6 \%$, in hybrid and intestinal, respectively). Within cases with intestinal immunophenotype, dysplasia was graded as low in most cases $(86.4 \%)(p=0.001)$. Gastric immunophenotype encompassed cases classified by conventional histology as gastric $(72.0 \%)$ and intestinal (28.0\%); hybrid immunophenotype encompassed cases classified by histology as gastric $(35.3 \%)$ and intestinal (64.7\%); and all cases of the intestinal immunophenotype displayed features of the adenomatous/intestinal histotype $(p<0.0001)$.

Table 5 shows the relationship between the presence of the coexistent intramucosal adenocarcinoma and the features of dysplasia (grade, histotype, and immunophenotype). In 20 of 64 cases (31.3\%), there was a coexistent carcinoma at the periphery of the dysplastic lesions, the latter displaying the following features: high grade (75.0\%; $\quad p=0.001) ; \quad$ gastric histotype $(60.0 \%$; $p=0.024)$; gastric immunophenotype $(65.0 \%, 20.0 \%$, and $15.0 \%$ for gastric, hybrid, and intestinal 
Fig. 2 Immunophenotypes of gastric dysplasia. Gastric immunophenotype displaying foveolar and pyloric/gastric histotype (a), high expression of MUC5AC (c) and MUC6 (e), and lack of expression of MUC2 (g) and CD10 (i). Intestinal immunophenotype displaying adenomatous/intestinal histotype (b), lack of expression of MUC5AC (d) and MUC6 (f), and expression of MUC2 (h) (inset MUC2 is expressed in goblet cells) and CD10 (j) (inset CD10 is exhibited at the apical pole of dysplastic cells). a,

b $\mathrm{H} \& \mathrm{E}$; c-

j immunohistochemical staining (IHC). $\times 100$
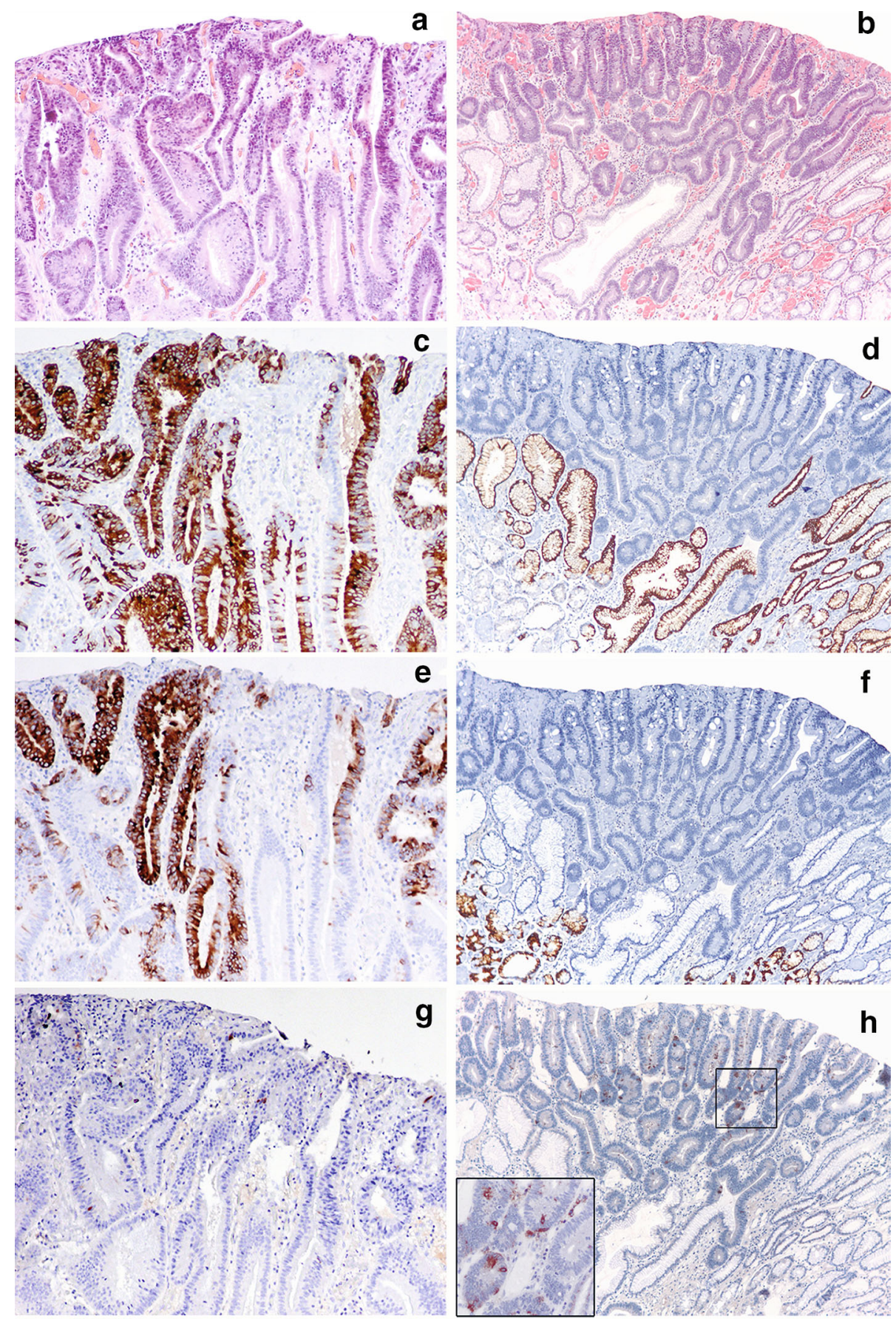

g
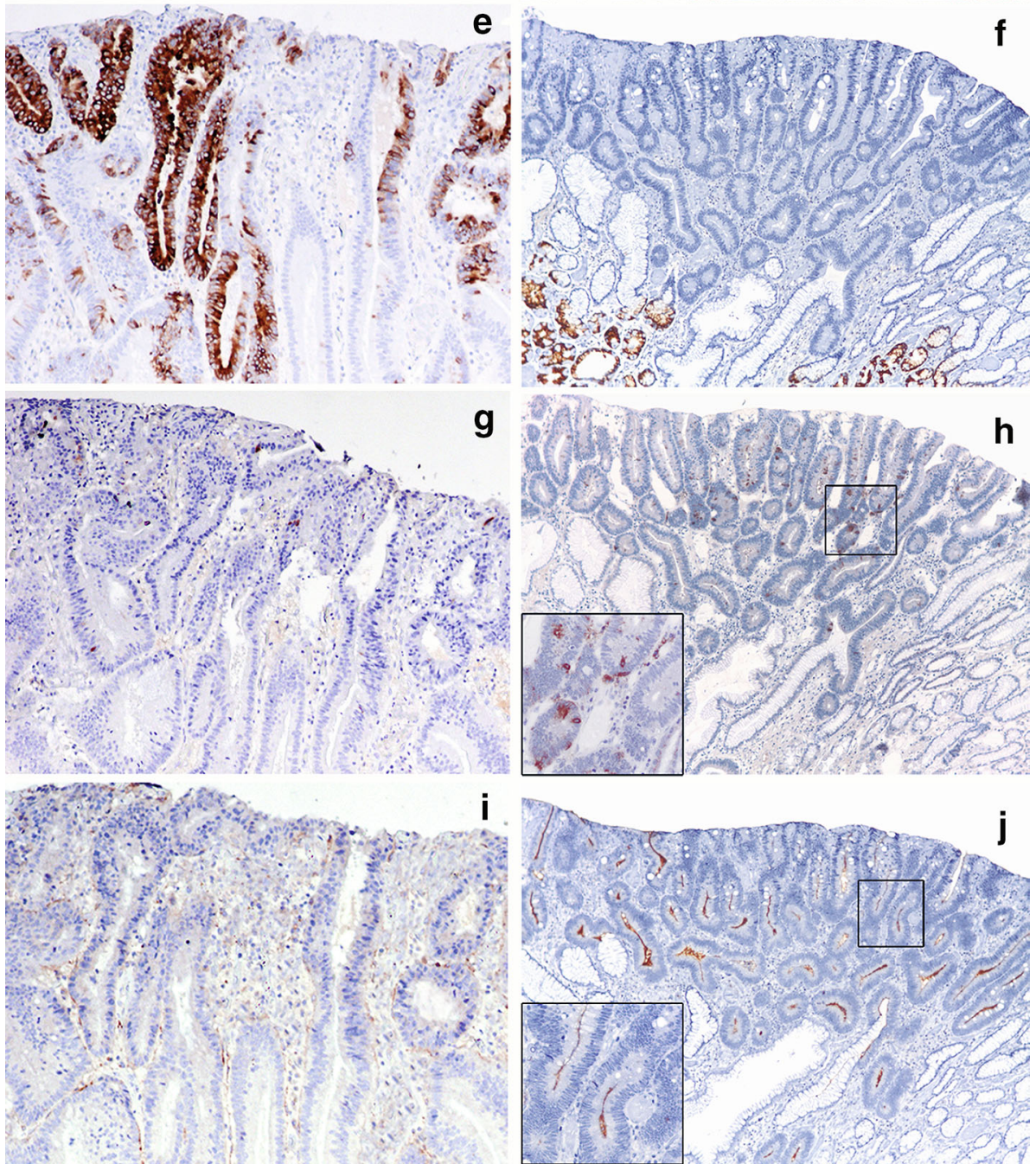
Table 3 Expression of the different markers according to the three immunophenotypes of gastric dysplasia

\begin{tabular}{|c|c|c|c|c|}
\hline & \multicolumn{3}{|c|}{ Immunophenotype } & \multirow[t]{2}{*}{$p$ value } \\
\hline & Gastric & Hybrid & Intestinal & \\
\hline \multicolumn{5}{|c|}{ MUC2 (\%) } \\
\hline$<5$ & $25(100 \%)$ & $10(58.8 \%)$ & $14(63.6 \%)$ & \\
\hline$\geq 5$ & 0 & $7(41.2 \%)$ & $8(36.4 \%)$ & 0.002 \\
\hline \multicolumn{5}{|c|}{ MUC5AC (\%) } \\
\hline$<5$ & $3(12.0 \%)$ & $8(47.1 \%)$ & $22(100 \%)$ & \\
\hline$\geq 5$ & $22(88.0 \%)$ & $9(52.9 \%)$ & 0 & 0.000 \\
\hline \multicolumn{5}{|c|}{$\operatorname{MUC6}^{\mathrm{a}}(\%)$} \\
\hline$<5$ & $2(8.7 \%)$ & $1(5.9 \%)$ & $22(100 \%)$ & \\
\hline$\geq 5$ & $21(91.3 \%)$ & $16(94.1 \%)$ & 0 & 0.000 \\
\hline \multicolumn{5}{|c|}{ CD10 (\%) } \\
\hline$<5$ & $24(96.0 \%)$ & $3(17.6 \%)$ & $2(9.1 \%)$ & \\
\hline$\geq 5$ & $1(4.0 \%)$ & $14(82.4 \%)$ & $20(90.9 \%)$ & 0.000 \\
\hline \multicolumn{5}{|c|}{ CDX2 $(\%)$} \\
\hline$<25$ & $5(20.0 \%)$ & 0 & 0 & \\
\hline$\geq 25$ & $20(80.0 \%)$ & $17(100 \%)$ & $22(100 \%)$ & 0.015 \\
\hline \multicolumn{5}{|c|}{ Ki-67 (\%) } \\
\hline$<50$ & $4(16.0 \%)$ & $1(5.9 \%)$ & $8(36.4 \%)$ & \\
\hline$\geq 50$ & $21(84.0 \%)$ & $16(94.1 \%)$ & $14(63.6 \%)$ & 0.050 \\
\hline \multicolumn{5}{|c|}{ TP53 (\%) } \\
\hline$<50$ & $21(84.0 \%)$ & $8(47.1 \%)$ & $13(59.1 \%)$ & \\
\hline$\geq 50$ & $4(16.0 \%)$ & $9(52.9 \%)$ & $9(40.9 \%)$ & 0.034 \\
\hline \multicolumn{5}{|c|}{ Synaptophysin (\%) } \\
\hline$<5$ & $17(68.0 \%)$ & $7(41.2 \%)$ & $4(18.2 \%)$ & \\
\hline$\geq 5$ & $8(32.0 \%)$ & $10(58.8 \%)$ & $18(81.8 \%)$ & 0.003 \\
\hline \multicolumn{5}{|c|}{ Chromogranin $^{\mathrm{a}}(\%)$} \\
\hline$<5$ & $18(72.0 \%)$ & $5(29.4 \%)$ & $1(4.8 \%)$ & \\
\hline$\geq 5$ & $7(28.0 \%)$ & $12(70.6 \%)$ & $20(95.2 \%)$ & 0.000 \\
\hline
\end{tabular}

a Missing data for MUC6 ( 2 cases) and chromogranin (1 case)

immunophenotypes, respectively; $p=0.013$ ). Gastric dysplasia at the periphery of invasive carcinoma, when compared with gastric dysplasia in the absence of invasive carcinoma, displayed a significantly lower frequency of expression of synaptophysin and chromogranin (30.0\% and $40.0 \% ; p=0.006$ and $p=0.025$, respectively).

\section{Discussion}

Gastric carcinogenesis is a complex process, requiring the elucidation of putative distinct pathways. According to the so-called Correa [4] model, gastric carcinogenesis is a multistep and multifactorial process that, in many cases, appears to involve a progression from normal mucosa, through chronic atrophic gastritis and intestinal metaplasia, to dysplasia and invasive carcinoma. However, evidence from the literature points to the possible existence of

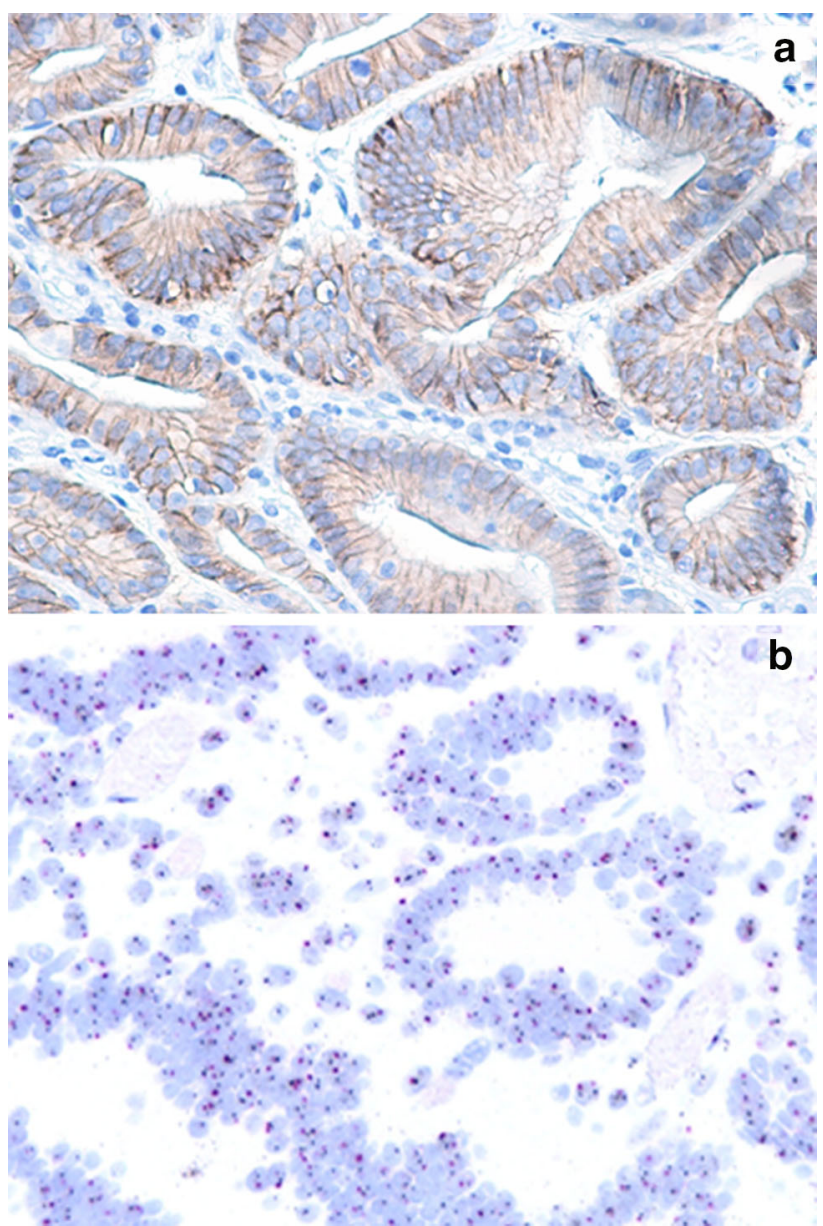

Fig. 3 Immunoexpression and amplification of HER2 in gastric dysplasia: weak/moderate immunoexpression of HER2 at the cell membrane, scored as $2+(\mathrm{IHC}, \times 100)($ a) and HER2 amplification evaluated by silver-enhanced in situ hybridization (SISH), $\times 100(\mathbf{b})$

Table 4 Relationship between immunophenotype and the histotype and grade of dysplasia

\begin{tabular}{lrrlll}
\hline & \multicolumn{2}{l}{ Immunophenotype } & \multirow{2}{*}{$p$ value } \\
\cline { 2 - 4 } & Gastric & Hybrid & Intestinal & \\
\hline Histotype & & & & \\
Gastric & $18(72.0 \%)$ & $6(35.3 \%)$ & 0 & 0.000 \\
Intestinal & $7(28.0 \%)$ & $11(64.7 \%)$ & $22(100 \%)$ & \\
Grade & & & & \\
Low grade & $8(32.0 \%)$ & $9(52.9 \%)$ & $19(86.4 \%)$ & \\
High grade & $17(68.0 \%)$ & $8(47.1 \%)$ & $3(13.6 \%)$ & 0.001 \\
\hline
\end{tabular}

alternative pathways in which intestinal metaplasia may not be involved. Evidence stems mainly from the study of tiny early GCs arising in nonmetaplastic mucosa, as described by Japanese authors $[14,15]$ as well as the studies of the expression of markers of gastric differentiation in dysplasia and gastric adenocarcinoma [7, 10, 16, 
Table 5 Comparison of the features of gastric dysplasia as isolated lesion or at the periphery of intramucosal gastric adenocarcinoma

\begin{tabular}{llrl}
\hline & \multicolumn{2}{l}{ Adenocarcinoma } & \multirow{2}{*}{$p$ value } \\
\cline { 2 - 3 } & Absent & Present & \\
\hline Grade of dysplasia & & & \\
$\quad$ Low grade & $31(70.5 \%)$ & $5(25.0 \%)$ & \\
$\quad$ High grade & $13(29.5 \%)$ & $15(75.0 \%)$ & 0.001 \\
Histotype & & & \\
Gastric & $12(27.3 \%)$ & $12(60.0 \%)$ & \\
Intestinal & $32(72.7 \%)$ & $8(40.0 \%)$ & 0.024 \\
Immunophenotype & & & \\
Gastric & $12(27.3 \%)$ & $13(65.0 \%)$ & \\
Hybrid & $13(29.5 \%)$ & $4(20.0 \%)$ & \\
Intestinal & $19(43.2 \%)$ & $3(15.0 \%)$ & 0.013 \\
Synaptophysin $(\%)$ & & & \\
$<5$ & $14(31.8 \%)$ & $14(70.0 \%)$ & \\
$\geq 5$ & $30(68.2 \%)$ & $6(30.0 \%)$ & 0.006 \\
Chromogranin ${ }^{\mathrm{a}}(\%)$ & & & \\
$<5$ & $12(27.9 \%)$ & $12(60.0 \%)$ & \\
$\geq 5$ & $31(72.1 \%)$ & $8(40.0 \%)$ & 0.025 \\
\hline
\end{tabular}

${ }^{a}$ Missing data for chromogranin (1 case)

17]. The latter demonstrate that both types of lesions may express, predominantly or exclusively, markers of gastric differentiation, raising the possibility of an origin in native gastric mucosa, rather than in intestinal metaplastic lesions. The role of spasmolytic polypeptide-expressing metaplasia (SPEM) in the pathogenesis of the lesions with gastric immunophenotype remains to be elucidated. Other evidence stems from hereditary gastric cancer models [hereditary diffuse gastric cancer and gastric adenocarcinoma and proximal polyposis of the stomach (HDGC and GAPPS)] in which GC, diffuse/poorly cohesive type, and intestinal/tubular type, respectively, originate in nonmetaplastic gastric epithelium (fundic gland polyps in the case of GAPPS) [18, 19].

Our study provides additional evidence in favor of de novo neoplastic transformation from native gastric mucosa (37.9\% of the dysplastic lesions displayed "pure" gastric immunophenotype).

Another relevant issue is the risk of malignant transformation of the different types of gastric dysplasia. Our results show that within the group of cases immunophenotyped as gastric, the majority were classified as highgrade dysplasia $(68.0 \% ; p=0.001)$. At variance, within cases immunophenotyped as intestinal, low-grade dysplasia was the most frequent $(86.4 \% ; p=0.001)$. These findings are in keeping with those recently reported by Nishimura et al. [14], but differ from the results reported by Abraham et al. [20]; the latter showed that intestinal-type adenomas were more likely than gastric-type adenomas to display high-grade dysplasia and adenocarcinoma in the polyps. A major difference from this study is that although the series studied by Abraham et al. [20] is composed of polypoid adenomas, our series is constituted by nonpolypoid dysplasia, not including polypoid/protruded lesions.

HER2 amplification was observed in three cases, immunophenotyped as gastric or hybrid. These findings show that HER2 amplification may be an early event in gastric carcinogenesis, as observed by Fassan et al. [21].

The results herein obtained in dysplasia with gastric immunophenotype (higher frequency of high-grade lesions, expression and amplification of HER2) suggest that this type of dysplasia may be an important factor in gastric carcinogenesis.

The high frequency of cases with high proliferative index (Ki-67) in gastric and hybrid immunophenotypes ( $84.0 \%$ and $94.1 \%$, respectively) when compared with the intestinal immunophenotype $(63.6 \% ; p=0.05)$ is in keeping with the features of aggressiveness identified in dysplastic lesions with gastric differentiation. At variance with other studies [22, 23], we have not found a significant difference in the Ki- 67 proliferation index according to the grade of dysplasia.

In this study, we observed that the expression of CDX2 is correlated with the intestinal immunophenotype (100\% of the cases), in keeping with data previously reported [9]. In accordance with Park et al. [9], a decreased expression of CDX2 was observed in cases with gastric immunophenotype ( $80 \%$ of the cases; decreased intensity of immunoreactivity). However, there is controversy in the literature regarding the expression of $\mathrm{CDX} 2$ in gastric dysplasia, probably reflecting the lack of subtyping of dysplasia in the different studies [9, 24, 25].

In some cases with intestinal immunophenotype, small nests of neuroendocrine cells were observed, qualifying for neuroendocrine hyperplasia as reported in the literature $[26,27]$. It is likely that adenomatous/intestinal dysplasia and neuroendocrine hyperplasia both arise in the setting of chronic atrophic gastritis, as previously suggested in neuroendocrine hyperplasia within gastric hyperplastic polyps [28]. However, further studies are needed to elucidate the biological meaning of this event.

In the present study we observed that higher expression of TP53 significantly correlated with the intestinal immunophenotype $(p=0.034)$ and was also more frequently observed in high-grade dysplasia, although this association was not significant ( $p=0.070$; data not shown). In previous studies, an increased frequency of TP53 overexpression along the progress of gastric carcinogenesis was observed. However, in these studies the immunohistochemical subtyping of gastric dysplasia was not performed [29]. Kushima et al. [30] showed that the frequency of 
TP53 expression was significantly higher in intestinal-type adenomas than in gastric-type adenomas, in keeping with the present study, and was higher in high-grade dysplasia than in low-grade dysplasia, leading to the suggestion that TP53 alterations occur earlier in the carcinogenetic sequence along the intestinal rather than the gastric differentiation pathway [30].

Summing up, our results point to the existence of two major types of dysplasia in the stomach. The gastric immunophenotype is significantly associated with highgrade dysplasia $(p=0.001)$, high proliferative index $(\mathrm{Ki}-$ 67) $(p=0.050)$, and coexistence of intramucosal adenocarcinoma $(p=0.013)$. The intestinal immunophenotype was shown to be significantly associated with low-grade dysplasia $(p=0.001), \quad$ overexpression of TP53 $(p=0.034)$, and neuroendocrine markers $(p=0.003$ for synaptophysin and $p<0.0001$ for chromogranin).

Recently, gene expression profiling using mRNA consensus clustering has revealed three distinct gastric cancer subtypes: mesenchymal, proliferative, and metabolic [31]. The metabolic subtype is characterized by the expression of genes normally expressed in gastric mucosa, involved in metabolic processes and digestion, and the expression of trefoil peptides [31] that are coexpressed in normal mucosa of the stomach with gastric mucins. These data are in keeping with the results of our previous studies showing the expression of trefoil peptides (and gastric mucins) in a subset of dysplastic and adenocarcinomatous lesions of the stomach [7, 16, 17], supporting the existence of a pathway of gastric carcinogenesis with gastric differentiation.

In face of the evidence we collected and that from the literature, we feel tempted to suggest that epithelial dysplasia of the stomach with gastric immunophenotype may represent the putative precursor lesion in a pathway of gastric carcinogenesis originated de novo from the native gastric mucosa, leading to a subset of glandular GCs with gastric differentiation.

Acknowledgments The authors thank Bárbara Gomes, Armando Castro, Armindo Pereira, and Manuel Moutinho (Centro Hospitalar de São João), Bárbara Sousa (IPATIMUP), and Franclim Ribeiro (Roche Diagnostics Portugal), for their technical support. This study was partly supported by a grant from the Portuguese affiliate of Hoffmann-La Roche AG (Roche Pharmaceuticals \& Roche Diagnostics, Portugal).

Conflict of interest The authors have no competing interest to declare.

\section{References}

1. Ferlay J, Shin HR, Bray F, Forman D, Mathers C, Parkin DM. Estimates of worldwide burden of cancer in 2008: GLOBOCAN 2008. Int J Cancer. 2010;127(12):2893-917.
2. Lauren P. The two histological main types of gastric carcinoma: diffuse and so-called intestinal-type carcinoma. An attempt at a histo-clinical classification. Acta Pathol Microbiol Scand. 1965;64:31-49.

3. Lauwers GY, Carneiro F, Graham DY, Curado MP, Franceschi S, Montgomery E, et al. Gastric carcinoma. In: Bosman FT, Carneiro F, Hruban RH, Theise ND, editors. WHO classification of tumours of the digestive system. 4th ed. Lyon: IARC; 2010. p. $48-68$.

4. Correa P. A human model of gastric carcinogenesis. Cancer Res. 1988;48(13):3554-60.

5. Rugge M, Cassaro M, Di Mario F, Leo G, Leandro G, Russo VM, et al. The long term outcome of gastric non-invasive neoplasia. Gut. 2003;52(8):1111-6.

6. Park SY, Jeon SW, Jung MK, Cho CM, Tak WY, Kweon YO, et al. Long-term follow-up study of gastric intraepithelial neoplasias: progression from low-grade dysplasia to invasive carcinoma. Eur J Gastroenterol Hepatol. 2008;20(10):966-70.

7. Nogueira AM, Machado JC, Carneiro F, Reis CA, Gott P, Sobrinho-Simoes M. Patterns of expression of trefoil peptides and mucins in gastric polyps with and without malignant transformation. J Pathol. 1999;187(5):541-8.

8. Park do Y, Srivastava A, Kim GH, Mino-Kenudson M, Deshpande V, Zukerberg LR, et al. Adenomatous and foveolar gastric dysplasia: distinct patterns of mucin expression and background intestinal metaplasia. Am J Surg Pathol. 2008;32(4):524-33.

9. Park do Y, Srivastava A, Kim GH, Mino-Kenudson M, Deshpande $\mathrm{V}$, Zukerberg LR, et al. CDX2 expression in the intestinaltype gastric epithelial neoplasia: frequency and significance. Mod Pathol. 2010;23(1):54-61.

10. Tsukashita S, Kushima R, Bamba M, Sugihara H, Hattori T. MUC gene expression and histogenesis of adenocarcinoma of the stomach. Int J Cancer. 2001;94(2):166-70.

11. The Paris endoscopic classification of superficial neoplastic lesions: esophagus, stomach, and colon: November 30 to December 1, 2002. Gastrointest Endosc. 2003;58(6 Suppl):S3S43.

12. Rüschoff J, Hanna W, Bilous M, Hofmann M, Osamura RY, Penault-Llorca F, et al. HER2 testing in gastric cancer: a practical approach. Mod Pathol. 2012;25(5):637-50.

13. Fassan M, Simbolo M, Bria E, Mafficini A, Pilotto S, Capelli P, et al. High-throughput mutation profiling identifies novel molecular dysregulation in high-grade intraepithelial neoplasia and early gastric cancers. Gastric Cancer. 2014;17(3):442-9.

14. Nishimura R, Mukaisho K, Yamamoto H, Sonoda A, Andoh A, Fujiyama $\mathrm{Y}$, et al. Precursor-derived versus de-novo carcinogenesis depends on lineage-specific mucin phenotypes of intramucosal gland-forming gastric neoplasms. Histopathology (Oxf). 2013;63(5):616-29.

15. Kushima R, Vieth M, Borchard F, Stolte M, Mukaisho K, Hattori T. Gastric-type well-differentiated adenocarcinoma and pyloric gland adenoma of the stomach. Gastric Cancer. 2006;9(3): $177-84$.

16. Machado JC, Carneiro F, Blin N, Sobrinho-Simões M. Pattern of $\mathrm{pS} 2$ protein expression in premalignant and malignant lesions of gastric mucosa. Eur J Cancer Prev. 1996;5(3):169-80.

17. Machado JC, Nogueira AM, Carneiro F, Reis CA, SobrinhoSimoes M. Gastric carcinoma exhibits distinct types of cell differentiation: an immunohistochemical study of trefoil peptides (TFF1 and TFF2) and mucins (MUC1, MUC2, MUC5AC, and MUC6). J Pathol. 2000;190(4):437-43.

18. Carneiro F, Charlton A, Huntsman DG. Hereditary diffuse gastric cancer. In: Bosman FT, Carneiro F, Hruban RH, Theise ND, editors. WHO classification of tumours of the digestive system. 4th ed. Lyon: IARC; 2010. p. 59-63. 
19. Worthley DL, Phillips KD, Wayte N, Schrader KA, Healey S, Kaurah $\mathrm{P}$, et al. Gastric adenocarcinoma and proximal polyposis of the stomach (GAPPS): a new autosomal dominant syndrome. Gut. 2012;61(5):774-9.

20. Abraham SC, Montgomery EA, Singh VK, Yardley JH, Wu TT. Gastric adenomas: intestinal-type and gastric-type adenomas differ in the risk of adenocarcinoma and presence of background mucosal pathology. Am J Surg Pathol. 2002;26(10):1276-85.

21. Fassan M, Mastracci L, Grillo F, Zagonel V, Bruno S, Battaglia $\mathrm{G}$, et al. Early HER2 dysregulation in gastric and oesophageal carcinogenesis. Histopathology (Oxf). 2012;61(5):769-76.

22. Sugai T, Inomata M, Uesugi N, Jiao YF, Endoh M, Orii S, et al. Analysis of mucin, p53 protein and $\mathrm{Ki}-67$ expressions in gastric differentiated-type intramucosal neoplastic lesions obtained from endoscopic mucosal resection samples: a proposal for a new classification of intramucosal neoplastic lesions based on nuclear atypia. Pathol Int. 2004;54(6):425-35.

23. Dong B, Xie YQ, Chen K, Wang T, Tang W, You WC, et al. (2005) Differences in biological features of gastric dysplasia, indefinite dysplasia, reactive hyperplasia and discriminant analysis of these lesions. World J Gastroenterol. 11(23):3595-600.

24. Rugge M, Ingravallo G, Farinati F, Russo VM, Zaninotto G, Alvisi V. Re: CDX2 homeotic gene expression in gastric noninvasive neoplasia. Am J Surg Pathol. 2004;28(6):834-835 (author reply 835).
25. Liu Q, Teh M, Ito K, Shah N, Ito Y, Yeoh KG. CDX2 expression is progressively decreased in human gastric intestinal metaplasia, dysplasia and cancer. Mod Pathol. 2007;20(12):1286-97.

26. Cockburn AN, Morgan CJ, Genta RM. Neuroendocrine proliferations of the stomach: a pragmatic approach for the perplexed pathologist. Adv Anat Pathol. 2013;20(3):148-57.

27. Lee SM, Ahn S, Lee YK, Jang KT, Park CK, Kim KM. Neuroendocrine tumor in gastric adenoma: a diagnostic pitfall mimicking invasive adenocarcinoma. Diagn Pathol. 2012;7:102.

28. Chetty R, Gill P, Mugon P, Shrimankar J, Hughes C. Gastric neuroendocrine cell hyperplasia and type 1 tumours occurring within gastric hyperplastic polyps. Virchows Arch. 2012;461(5): 483-7.

29. Rugge M, Shiao YH, Correa P, Baffa R, DiMario F. Immunohistochemical evidence of $\mathrm{p} 53$ overexpression in gastric epithelial dysplasia. Cancer Epidemiol Biomark Prev. 1992;1(7):551-4.

30. Kushima R, Muller W, Stolte M, Borchard F. Differential p53 protein expression in stomach adenomas of gastric and intestinal phenotypes: possible sequences of p53 alteration in stomach carcinogenesis. Virchows Arch. 1996;428(4-5):223-7.

31. Lei Z, Tan IB, Das K, Deng N, Zouridis H, Pattison S, et al. Identification of molecular subtypes of gastric cancer with different responses to PI3-kinase inhibitors and 5-fluorouracil. Gastroenterology. 2013;145(3):554-65. 